preferable. The most-discussed question at the meeting is likely to be about the use of $\mathrm{CO}_{2}$.

"People do still worry about $\mathrm{CO}_{2}$ and it's still almost certainly the most widely used method [for killing rodents]," says Huw Golledge, who studies the anaesthesia of lab animals at Newcastle University, UK. Golledge organized the meeting, which is backed by the London-based National Centre for the Replacement, Refinement and Reduction of Animals in Research. Its aim is to update a 2006 consensus document produced by international experts to give guidance to researchers working with animals.

$\mathrm{CO}_{2}$ is used to make rodents unconscious. They are then killed by either asphyxiating them with the gas, or by another method. But increasingly, studies suggest rodents find $\mathrm{CO}_{2}$ stressful.

Evidence for this comes mainly from 'aversion studies'. A key study by animal welfare researcher Daniel Weary's group at the University of British Columbia in Vancouver, Canada, shows that albino rats will move away from a dark compartment filling with $\mathrm{CO}_{2}$ into a brightly lit box, despite disliking bright lights. The study found that they were less likely to move away from isoflurane, also used in euthanasia (D. Wong et al. Biol. Lett. http://doi.org/ ncv; 2012).

Other evidence is contradictory (H. Valentine et al. J. Am. Assoc. Lab. Anim. Sci. 51, 50-57; 2012), but Weary is firm in his beliefs. "Our own results indicate $\mathrm{CO}_{2}$ is highly aversive," he says.

There are also question marks over physical methods. Performed perfectly with animals accustomed to being handled, cervical dislocation may be the best method, but it may not be practical for killing large numbers of rodents.

The issues are even more uncertain for the new animal models that scientists are pursuing. For example, a huge increase in the use of zebrafish has put them on the meeting's agenda. Although much progress has been made with lab rodents, says Weary, "there's been much less work on fish welfare in general".

Widely used guidelines on animal euthanasia from the American Veterinary Medical Association (AVMA) in Schaumburg, Illinois, were updated earlier this year, in part to adapt to changes in the animals used in labs, with zebrafish guidance one of the additions. The association says it expects the lab animals section of these guidelines to continue to expand.

Some of this guidance comes with regulatory teeth. Later this year, the US National Institutes of Health, which funds biomedical research, says that it expects "full implementation" of the AVMA guidelines, with previously approved projects reviewed using them.

Regulation is also driving more unusual animals onto the agenda. New legislation on the treatment of laboratory animals is currently being incorporated into the laws of European Union member states and will cover cephalopods, in some nations for the first time (see Nature http://doi.org/fk65pb; 2011).

OPTICS

\title{
Squeezed light mutes quantum noise
}

\section{Silicon zip reduces energy fluctuations in laser beams to improve sensitivity of optical motion sensors.}

\section{BY DEVIN POWELL}

$\mathrm{O}$ skar Painter spends his time carving silicon blocks into shapes that interact with light in strange ways. The latest of these - what Painter calls "Legos for adults" - squeezes the light from laser beams to push the limits of what they can measure.

By eliminating some of the noise caused by quantum effects, researchers can use squeezed light to illuminate movements too small to see with normal light. Painter's silicon sculpture, built on a microchip, could boost the sensitivity of sensors that use lasers to monitor motion, such as the gyroscopes that keep track of an aircraft's orientation.

"We have an opportunity to push the performance of these sensors by orders of magnitude," says Painter, an applied physicist at the California Institute of Technology in Pasadena, whose team reports how it squeezes light on page 185 (ref. 1).

All light is plagued by quantum noise, especially at the low powers typically required by sensors. These energy fluctuations blur the defined peaks of classical light waves, fundamentally limiting the precision of measurements.

Squeezing the light can suppress some noise, but Heisenberg's uncertainty principle demands a trade-off. A squeeze that reduces noise in one dimension - the height of a light wave's peaks, for instance - must be balanced by a stretch that adds noise in another, such as the distance between the peaks. Researchers therefore have to match the direction of the squeezing to the direction of the measurement.

Efforts to put light-squeezing to use have so far focused on gravitational-wave detectors, which search for faint ripples in spacetime by timing laser beams as they bounce between mirrors 4 kilometres apart. Passing ripples should stretch or compress the laser beams ever so slightly. But measurements with normal laser light are limited by quantum noise, and have so far failed to detect any disturbances attributable to gravitational waves.

Hoping to improve the next generation of measurements, researchers at the Laser Interferometer Gravitational-Wave Observatory

(LIGO) in Hanford, Washington, added a dose of squeezed light by passing laser light through a crystal. In July, they reported that they had achieved a sensitivity better than the standard limit imposed by quantum noise ${ }^{2}$. This represents a step towards the ultimate goal of doubling LIGO's sensitivity, says team member Nergis Mavalvala, a physicist at the Massachusetts Institute of Technology in Cambridge. "We have to work hard to strip the noise out of the light," she says.

Painter's silicon device potentially offers a simpler way to squeeze light, although only at frequencies too high to be useful for gravitational-wave detectors. The device looks

"We have to
work hard
to strip the
noise out of the
light."
like a zip; photons bouncing around between its two arms push them apart with a force dictated by the amount of noise in the light. As the size of the gap changes, the zip tunes the frequency of the light - just as a finger sliding along a guitar string changes the pitch of the sound produced - and squeezes out some of the fluctuations.

The prototype tends to leak light, so it can suppress only about $5 \%$ of the noise. "The absolute level of squeezing is relatively low," says Warwick Bowen, a physicist at the University of Queensland in Brisbane, Australia. Painter says he will next be working with higher-quality zips, which could cut out as much as $90 \%$ of the noise.

But he will have some competition. A team at JILA in Boulder, Colorado, a joint institute of the University of Colorado and the US National Institute of Standards and Technology, has already created a vibrating silicon nitride membrane that boasts a $32 \%$ reduction in noise. JILA physicist Cindy Regal and her colleagues will report their work in a paper under review at Physical Review X (ref. 3). "It has been technically challenging to get to this regime," says Regal.

1. Safavi-Naeini, A. H. et al. Nature 500, 185-189 (2013).

2. The LIGO Scientific Collaboration Nature Photon. 7,613-619 (2013).

3. Purdy, T. P., Yu, P.-L., Peterson, R. W., Kampel, N. S. \& Regal, C. A. Preprint at http://arxiv.org/ abs/1306.1268 (2013). 